$$
\begin{gathered}
x-x+19 \\
1 N-89 \\
1-19
\end{gathered}
$$

\title{
Velocity-Resolved Observations of Water in Comet Halley
}

Harold P. Larson, D. Scott Davis, Michael J. Mumma, and Harold A. Weaver

(HASA-TH-88367) VELOCITI-EESCIVED CESERVATICNS CP HATER IH CCHET HALLEY (NASA) $19 \mathrm{p}$ avail: ITIS EC $102 / 1 \mathrm{~F}$ 101 CSCL O3A

Unclas H1/89 0070058

September 1986

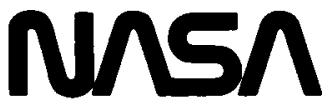

National Aeronautics and Space Administration
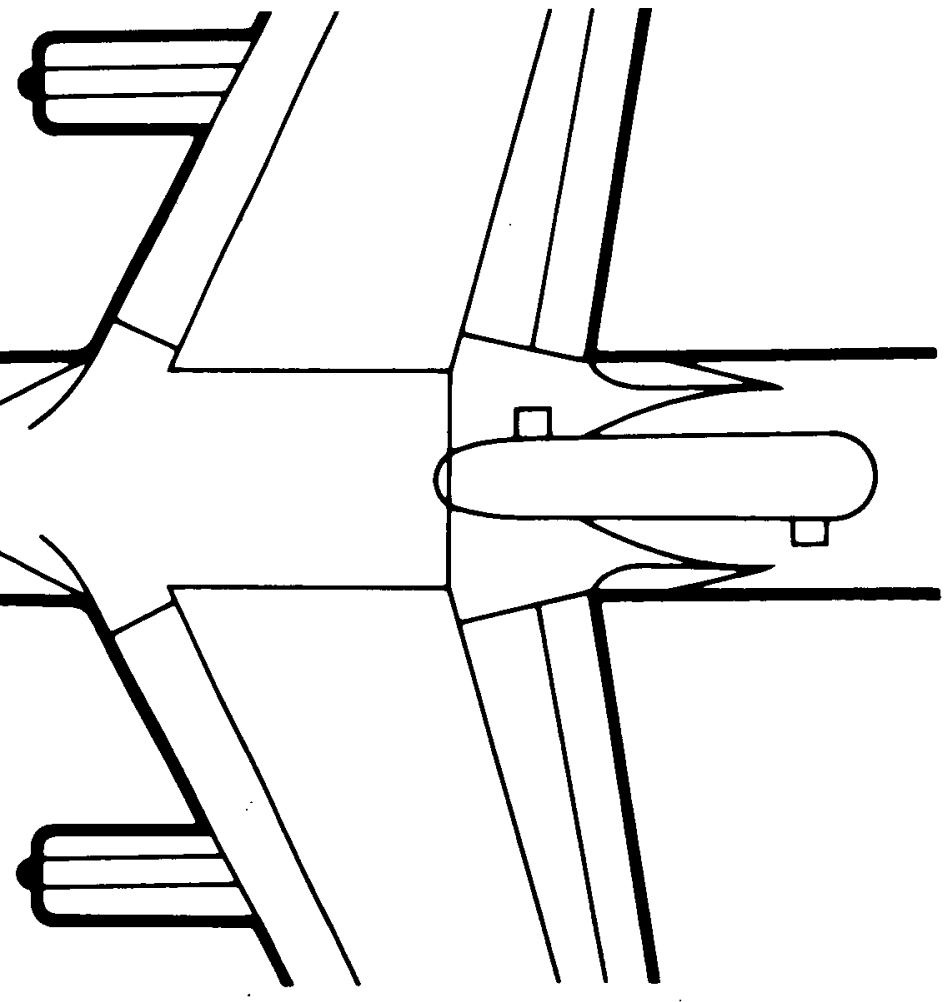


\section{Velocity-Resolved Observations of Water in Comet Halley}

Harold P. Larson, Lunar and Planetary Observatory, University of Arizona, Tucson, Arizona D. Scott Davis, Steward Observatory, University of Arizona, Tucson, Arizona Michael J. Mumma, Goddard Space Flight Center, Greenbelt, Maryland Harold A. Weaver, The Center for Astrophysical Sciences, Johns Hopkins University, Baltimore, Maryland

\section{N/Sก}

National Aeronautics and

Space Administration 
ABSTRACT

High resolution $\left(\lambda / \Delta \lambda \approx 3 \times 10^{5}\right)$ near-infrared observations of $\mathrm{H}_{2} \mathrm{O}$ emission from Comet Halley were acquired at the time of maximum postperihelion geocentric Doppler shift. The observed widths and absolute positions of the $\mathrm{H}_{2} \mathrm{O}$ line profiles reveal characteristics of the molecular velocity field in the coma. These results support $\mathrm{H}_{2} \mathrm{O}$ outflow from a Sun-1it hemisphere or the entire nucleus, but not from a single, narrow jet emanating from the nucleus. The measured pre- and post-perihelion outflow velocities were $0.9 \pm 0.2$ and $1.4 \pm 0.2 \mathrm{~km} \mathrm{~s}^{-1}$, respectively. Temporal variations in the kinematic properties of the outflow were inferred from changes in the spectral line shapes. These results are consistent with the release of $\mathrm{H}_{2} \mathrm{O}$ into the coma from multiple jets. 


\section{INTRODUCTION}

The detection of gaseous $\mathrm{H}_{2} \mathrm{O}$ in Comet Halley from the NASA Kuiper Airborne Observatory (KAO) convincingly established that $\mathrm{H}_{2} \mathrm{O}$ is a major volatile component of cometary matter (Mumma et al. 1986). That work also demonstrated that excitation of the $\mathrm{H}_{2} \mathrm{O} \quad v_{3}$ vibration-rotation band in the coma is dominated by infrared fluorescence, a mechanism that only recently received theoretical attention (see e.g. Weaver and Mumma 1984). The combination of a realistic infrared excitation model and high spectroscopic sensitivity for studying near-infrared cometary $\mathrm{H}_{2} \mathrm{O}$ bands from aircraft gives unprecedentedly detailed glimpses into other physical conditions in the coma. Weaver et al. (1986) used this diagnostic potential at moderate spectral resolution $\left(\lambda / \Delta \lambda \approx 3 \times 10^{3}\right)$ to study temporal activity and spatial variations in Comet Halley's $\mathrm{H}_{2} \mathrm{O}$ emission, and they correlated this information with other spectral data, including simultaneous observations of $\mathrm{OH}$ and continuum emission from the International Ultraviolet Explorer satellite (IUE). We present here very high resolution $\left(\lambda / \Delta \lambda \approx 3 \times 10^{5}\right)$ observations of $\mathrm{H}_{2} \mathrm{O}$ in Comet Halley. The positions and widths of the $\mathrm{H}_{2} \mathrm{O}$ emission line profiles reveal characteristics of the velocity field in the coma.

\section{OBSERVATIONS}

The high resolution observations were made aboard NASA's Kuiper Airborne Observatory on UT 22 March 1986 from 16:30 to 17:50 using the University of Arizona Fourier transform spectrometer (Davis et al. 1980). The KAO was based in Christchurch, New Zealand to optimize post-perihelion 
observations of Comet Halley. A cold filter limited the spectrometer's spectral bandpass from 3745 to $3830 \mathrm{~cm}^{-1}$. This interval included most of the transitions in the $\mathrm{H}_{2} \mathrm{O} v_{3}$ band that would be excited by infrared fluorescence. The angular field-of-view (41" diameter) centered on the cometary nucleus corresponded to a spatial field of $2.2 \times 10^{4} \mathrm{~km}$ diameter at the comet's geocentric distance $\Delta=0.75 \mathrm{a} . \mathrm{u}$. The flight altitude of the KAO was maintained at $12.4 \mathrm{~km}$ where the average line-of-sight residual terrestrial $\mathrm{H}_{2} \mathrm{O}$ abundance was about 10 prec. $\mu \mathrm{m} \mathrm{H}_{2} \mathrm{O}$. A lunar comparison spectrum was recorded on UT 24 March 1986 to evaluate atmospheric and instrumental transmission factors.

A portion of the comet's spectrum is displayed in Figure 1. Thirteen $\mathrm{H}_{2} \mathrm{O}$ lines were identified in the complete spectrum; these are listed in Table 1. The signal-to-noise (rms) ratio in the strongest observed line is about 80 . The theoretical unapodized instrumental resolution (Rayleigh criterion) is $0.014 \mathrm{~cm}^{-1}$, equivalent to a velocity width of 1.1 $\mathrm{km} \mathrm{s} \mathrm{s}^{-1}$ at $3800 \mathrm{~cm}^{-1}$. For comparison, the signal-to noise ratio in our pre-perihelion observations was about 40 and the instrumental velocity resolution was $3.2 \mathrm{~km} \mathrm{~s}^{-1}$ (Mumma et al. 1986). The improvement in our post-perihelion data is due in part to a smaller geocentric distance, but primarily it is a consequence of higher line intensities due to an increased $\mathrm{H}_{2} \mathrm{O}$ production rate (Weaver et al. 1986). The cometary $\mathrm{H}_{2} \mathrm{O}$ lines in Figure 1 are Doppler-shifted to shorter wavelengths because of the comet's geocentric velocity $₫ \approx-43 \mathrm{~km} \mathrm{~s}^{-1}$. This large value placed each cometary line on the far blue wing of the corresponding telluric $\mathrm{H}_{2} \mathrm{O}$ absorption (see Figure 2). The full profile of each cometary line was therefore recorded with minimum interference from terrestrial $\mathrm{H}_{2} \mathrm{O}$. This spectrum 
could not have been recorded at a ground-based facility because of complete obscuration of cometary features by telluric $\mathrm{H}_{2} \mathrm{O}$. Furthermore, nothing comparable was achieved from spaceborne infrared spectrometers because of their restriction to very low resolving powers $[\lambda / \Delta \lambda=40$ on the VEGA IKS experiment (Combes et a1. 1986); $\lambda / \Delta \lambda=70$ on the VEGA TKS experiment (Kras nopolsky et al. 1986)].

The cometary $\mathrm{H}_{2} \mathrm{O}$ line positions in Table 1 are expressed as absolute wavenumbers in vacuum. They were corrected for the refractive index of air under experimental conditions and for the finite sizes of the signal and reference beam apertures. To remove possible systematic error due to uncertainty associated with the actual wavelength of the spectrometer's reference laser, we compared the positions of isolated $\mathrm{H}_{2} \mathrm{O}$ absorption lines observed in the lunar comparison spectrum with interferometrically measured laboratory values. Small systematic differences, typically $+0.006 \mathrm{~cm}^{-1}$ or less, were fit to a low order polynomial correction function that was applied to both the lunar and cometary spectra. This procedure therefore tied the final absolute wavenumber calibration of the astronomical spectra to the best available laboratory measurements [Camy-Peyret et al. (1973), who claim an absolute accuracy better than $\pm 0.003 \mathrm{~cm}^{-1}$, or $\pm 0.24 \mathrm{~km} \mathrm{~s}^{-1}$ at $3800 \mathrm{~cm}^{-1}$ ].

The absolute calibration of the cometary $\mathrm{H}_{2} \mathrm{O}$ line intensities is not essential for this paper. We note, however, that our absolute intensities for the lines in Table 1 lead to a water production rate $\mathrm{Q}_{\mathrm{H}_{2} \mathrm{O}} \approx 1 \times 10^{30}$ $\operatorname{mol} \mathrm{s}^{-1}$. 
III. RESULTS

\section{a) Nucleocentric $\mathrm{H}_{2} \mathrm{O}$ Velocity}

Three velocities contribute to the observed positions of the cometary lines in Table 1: (1) the motion of the comet relative to the Earth (geocentric component); (2) the observatory's motion due to the Earth's rotation and the KAO's flight track (topocentric component); and (3) the net outflow velocity of $\mathrm{H}_{2} \mathrm{O}$ molecules from the nucleus (nucleocentric component). The geocentric velocity component was $-43.25 \mathrm{~km} \mathrm{~s}-1$ (Edberg 1986; negative velocities are directed towards the Earth). The topocentric velocity $v_{\mathrm{KAO}}=-0.25 \mathrm{~km} \mathrm{~s}^{-1}$ was calculated from the KAO flight log. Wavenumber corrections for these two velocity components were applied to each of the observed $\mathrm{H}_{2} \mathrm{O}$ lines. The calculated nucleocentric $\mathrm{H}_{2} \mathrm{O}$ line positions (see Table 1) should then differ from laboratory rest positions only if there is a net outflow of $\mathrm{H}_{2} \mathrm{O}$ molecules either towards or away from the Earth. The weighted average of these differences is $-0.21 \pm 0.12$ $\mathrm{km} \mathrm{s}^{-1}$. A similar treatment of our pre-perihelion data (Mumma et al. 1986) yields $-0.18 \pm 0.15 \mathrm{~km} \mathrm{~s}^{-1}$. Both measurements indicate that the $\mathrm{H}_{2} \mathrm{O}$ outflow distributions, averaged over the spectrometer's field-of-view, have no dominant velocity components along the Comet-Earth line-of-sight. The quoted $1 \sigma$ errors suggest that there may be small blue shifts to the distributions, but more conservative error estimates are consistent with no shifts.

These results are surprising. The maximum $\mathrm{H}_{2} \mathrm{O}$ outflow is expected to occur towards the Sun with possibly a small phase lag angle $\phi$ with respect to the sub-solar point because of nuclear rotation. The Suncomet-Earth angles at the time of our pre- and post-perihelion observations 
were $55^{\circ}$ and $66^{\circ}$, respectively. The viewing geometry would then have placed the maximum $\mathrm{H}_{2} \mathrm{O}$ outflow at angle $\theta=55^{\circ}-\phi$ with respect to the cometEarth line-of-sight at pre-perihelion and at $\theta=66^{\circ}+\phi$ at post-perihelion. We estimate that $\phi \approx-8^{\circ}$ from our nucleocentric velocity measurements. This value implies that (1) the $\mathrm{H}_{2} \mathrm{O}$ outflow actually was spatially restricted to a direction along or near the Sun-comet axis, or (2) the outflow distribution only appeared to be directed along this axis because of velocity averaging in the spectrometer's field-of-view. The first possibility is excluded for two reasons. First, if the outflow was restricted to a single, narrow jet with $\phi \approx-8^{\circ}$ and $v_{t} \approx 1 \mathrm{~km} \mathrm{~s}^{-1}$, the observed pre- and post-perihelion nucleocentric velocities should have been about $-0.5 \mathrm{~km} \mathrm{~s}^{-1}$, contrary to the observed values. Second, the natural width of the cometary lines for such an outflow would be rather narrow, contrary to the observed line widths which are discussed in the next section. The outflow must therefore be more widely dispersed. The above measurements do not uniquely constrain the spatial extent of the outflow, but they do require that the distribution of velocities in the spectrometer's fieldof-view produce very small or no net nucleocentric velocity components for both the pre- and post-perihelion viewing geometry. Several possible outflow distributions consistent with this constraint are discussed in the next section.

\section{b) The Gas Velocity Field in the Coma}

The outflow distribution will influence the observed $\mathrm{H}_{2} \mathrm{O}$ spectral line profiles at sufficiently high resolution. In general, the observed line profile is the convolution of the comet's velocity dispersion function 
(VDF) with the experimental instrumental line shape (ILS). The data in Figure 2 illustrate $\mathrm{H}_{2} \mathrm{O}$ line profiles from our high resolution spectra. The ILS for the pre-perihelion data was a sinc function distinguished by its very prominent side lobes; its width defined by the Rayleigh criterion was $3.2 \mathrm{~km} \mathrm{~s}^{-1}$. The observed cometary line profile is so similar to the ILS that the width of the VDF must be substantially less than $3.2 \mathrm{~km} \mathrm{~s}^{-1}$. The ILS for the higher resolution post-perihelion observations was also a sinc function (width=1.1 $\mathrm{km} \mathrm{s}^{-1}$ ). However, the observed cometary line profile has a width of $2.4 \mathrm{~km} \mathrm{~s}^{-1}$ and the side lobes are completely suppressed. The cometary line is therefore at least partially resolved, and the width of the VDF must be larger than that of the ILS. More stringent limits to the velocity width result from applying deconvolution analysis to the observed line profiles. We considered two simple distributions: (1) outflow into a Sun-facing hemisphere oriented approximately perpendicularly to the comet-Earth line-of-sight; and (2) a spherically symmetric outflow. Both allow projected velocity components from $-v_{t}$ to $+v_{t}$ along the lineof-sight without producing a net nucleocentric velocity shift. The shape of the VDF is determined by the actual distribution of velocities in the outflow. For this preliminary analysis we used rectangular functions (FWHM $=2 v_{t}$ ) to represent hemispherical or spherical uniform outflow. We convolved rectangular functions having different widths with the ILS and compared the calculated profiles with the observed cometary lines. We found that the maximum width compatible with the pre-perihelion line profile was $1.8 \pm 0.4 \mathrm{~km} \mathrm{~s}^{-1}$ and the minimum width required for the postperihelion data was $2.7 \pm 0.4 \mathrm{~km} \mathrm{~s}^{-1}$. The uncertainties in $v_{t}$ were obtained by propagating the 10 errors in the measured line widths through the 
numerical convolution. These measurements imply that the VDF's may have been different for our two high resolution spectra. This difference was confirmed in the following manner. The post-perihelion cometary line profile in Figure 2 was Fourier transformed at the lower resolution of the pre-perihelion data. It was clearly broader than either the theoretical ILS or the observed $\mathrm{H}_{2} \mathrm{O}$ line at pre-perihelion, and its side lobes were not fully developed. This comparison demonstrated independently that the natural width of the cometary $\mathrm{H}_{2} \mathrm{O}$ lines was larger during the post-perihelion observations. This difference may be due to changes in the shape of the VDF, to an increase in the value of $v_{t}$, or both. The observed line profiles could also be influenced by temporal changes in the comet's brightness. In particular, apodization (i.e broadening) of the profile would result if the comet's brightness decreased during data acquisition. However, we have no independent evidence to support this possibility, and it is equally probable that the comet could have brightened during the scan. For this preliminary analysis we interpret the observed line profiles in terms of rectangular VDF's differing only in width. Our estimates of $v_{t}$ become $0.9 \pm 0.2$ and $1.4 \pm 0.2 \mathrm{~km} \mathrm{~s}^{-1}$ for the pre- and post-perihelion spectra, respectively. These values can be compared to theoretical predictions of about $1 \mathrm{~km} \mathrm{~s}^{-1}$ and to the in situ measurement of $0.90 \pm 0.20 \mathrm{~km} \mathrm{~s}^{-1}$ from the mass spectrometer on Giotto (Krankowsky et al. 1986).

\section{SUMMARY}

Our spectroscopic measurements support the following description of molecular outflow from Comet Halley. 
1. Water appears to be widely dispersed around the nucleus. One plausible distribution is outflow into a presumably Sun-facing hemisphere, although spherically symmetric outflows cannot be excluded. The data are not compatible with outflow into any narrow solid angle.

2. The measured pre- and post-perihelion $\mathrm{H}_{2} \mathrm{O}$ terminal velocities were $0.9 \pm 0.2$ and $1.4 \pm 0.2 \mathrm{~km} \mathrm{~s}^{-1}$, respectively. Since they apply to nearly the same heliocentric distance of the comet $(\approx 1$ a.u. $)$, their difference may indicate an unexpected asymmetry in the dynamic properties of the pre- and post-perihelion outflows. However, the shape of the VDF must be constrained by a more detailed deconvolution analysis in order to pursue this interpretation.

3. The outflow exhibited temporal variations as evidenced by changes in the natural width of the observed spectral lines. This variability may be diagnostic of some kinematic property of the outflow, but more analysis is required to identify it. It is significant to note that the water production rates derived from these infrared spectra also showed strong temporal variability, and, in particular, they were significantly enhanced in the post-perihelion data (Weaver et al. 1986).

The above description is consistent both with general expectations concerning the release of volatile constituents from cometary matter and with more detailed results from other recent observations of Comet Halley. For example, direct images of Comet Halley from Giotto revealed multiple jets releasing material into most of the Sun-facing hemisphere (Keller et al. 1986). These jets plausibly sustain the field-averaged distribution deduced from our infrared spectra and they also account for the temporal variations that appear in many kinds of measurements of Comet Halley. We 
observed large, rapid variations in the $\mathrm{H}_{2} \mathrm{O}$ line intensities throughout our program of airborne observations of Comet Halley (Weaver et al. 1986), and others noted strong outbursts on time scales of a few hours [e.g. ground-based images of dust outbursts (Sekanina and Larson 1986); dust and gas spectrophotometry from IUE (Festou et al. 1986)]. This activity emphasizes the need to develop more realistic physical models of comets. We have demonstrated that remote infrared spectroscopic measurements can contribute to this task by providing measurements of kinematic conditions in the coma. This capability can be refined and applied to other comets which will not be as intensively studied as was Comet Halley.

Acknowledgements: It is a pleasure to commend the staff of the NASA Kuiper Airborne Observatory for making the Comet Halley expedition to New Zealand look so routine. Our success was a direct measure of their attention to detail under less-than-ideal field conditions. This research was supported by NASA Grants NAG2-206 to the University of Arizona and NAG2-356 to the Johns Hopkins University, and by RTOP's 188-41-55, 19641-54, and 352-02-03 to the Goddard Space Flight Center. 


\section{REFERENCES}

Camy-Peyret, C., Flaud, J.M., Guelachvili, G., and Amiot, C. 1973, Molec. Phys., 26, 825 .

Combes, M., et al. 1986, Nature, 321, 266.

Davis, D.S., Larson, H.P., Williams, M., Michel, G., and Connes, P. 1980, App1. Optics, 19, 4138.

Edberg, S.J. (ed.) 1986, International Halley Watch Newsletter, 8, 51.

Festou, M.C., et al. 1986, Nature, 321, 361.

Keller, H.U., et al. 1986, Nature, 321, 320.

Krankowsky, D., et al. 1986, Nature, 321, 326.

Krasnopolsky, V.A., et al. 1986, Nature, 321, 269.

Mumma, M.J., Weaver, H.A., Larson, H.P., Davis, D.S., and Williams, M. 1986, Science, 232, 1523.

Sekanina, Z., and Larson, S.M. 1986, Nature, 321, 357.

Weaver, H.A., and Mumma, M.J. 1984, Ap.J., 226, 782.

Weaver, H.A., Mumna, M.J., Larson, H.P., and Davis, D.S. 1986, Nature, submitted. 


\section{FIGURE CAPTIONS}

Figure 1. A portion of the velocity-resolved airborne spectrum of $\mathrm{H}_{2} \mathrm{O}$ in Comet Halley. The line intensities are uncorrected for instrumental and atmospheric transmission factors. The accompanying lunar comparison spectrum indicates residual telluric $\mathrm{H}_{2} \mathrm{O}$ absorptions. The plus signs designate cometary $\mathrm{H}_{2} \mathrm{O}$ lines assigned to the 001-000 vibration band, and the crosses indicate $\mathrm{H}_{2} \mathrm{O}$ transitions assigned to the "hot" 011-010 band. The quantum numbers of the upper and lower states accompany each cometary line. The corresponding transitions in the 001-000 band in absorption in the Earth's atmosphere are marked with arrows on the lunar wavenumber axis. The cometary observations were scheduled at nearly maximum geocentric Doppler shift, thus accounting for the wide separation of the cometary and telluric features. The "hot" lines in the comet's spectrum do not appear in the lunar spectrum; they are almost certainly pumped in the 011-000 band followed by fluorescence in the 011-010 band. Some strong $\mathrm{H}_{2} \mathrm{O}$ absorption lines in the 001-000 band in the lunar spectrum without accompanying, Doppler-shifted cometary emission lines are marked with their quantum numbers. These features arise from higher levels that are rotationally relaxed in the cometary coma. Telluric lines belonging to the 100-000 band are marked with asterisks and are not expected to be present in the comet's spectrum.

Figure 2. Comparison of pre- and post-perihelion $\mathrm{H}_{2} \mathrm{O}$ emission line profiles from Comet Halley. The post-perihelion data are characterized by higher SNR and higher instrumental resolution. The pre-perihelion line 
profile is unresolved. The structure at the base of the profile is due to the side lobes of the ILS. The post-perihelion line profile lacks these side lobes because the natural velocity width of the cometary line is at least partially resolved. The ordinate scale indicates relative instrumental transmission in the lunar spectrum. The cometary lines are arbitrarily scaled to the same height for direct comparison of their profiles. 


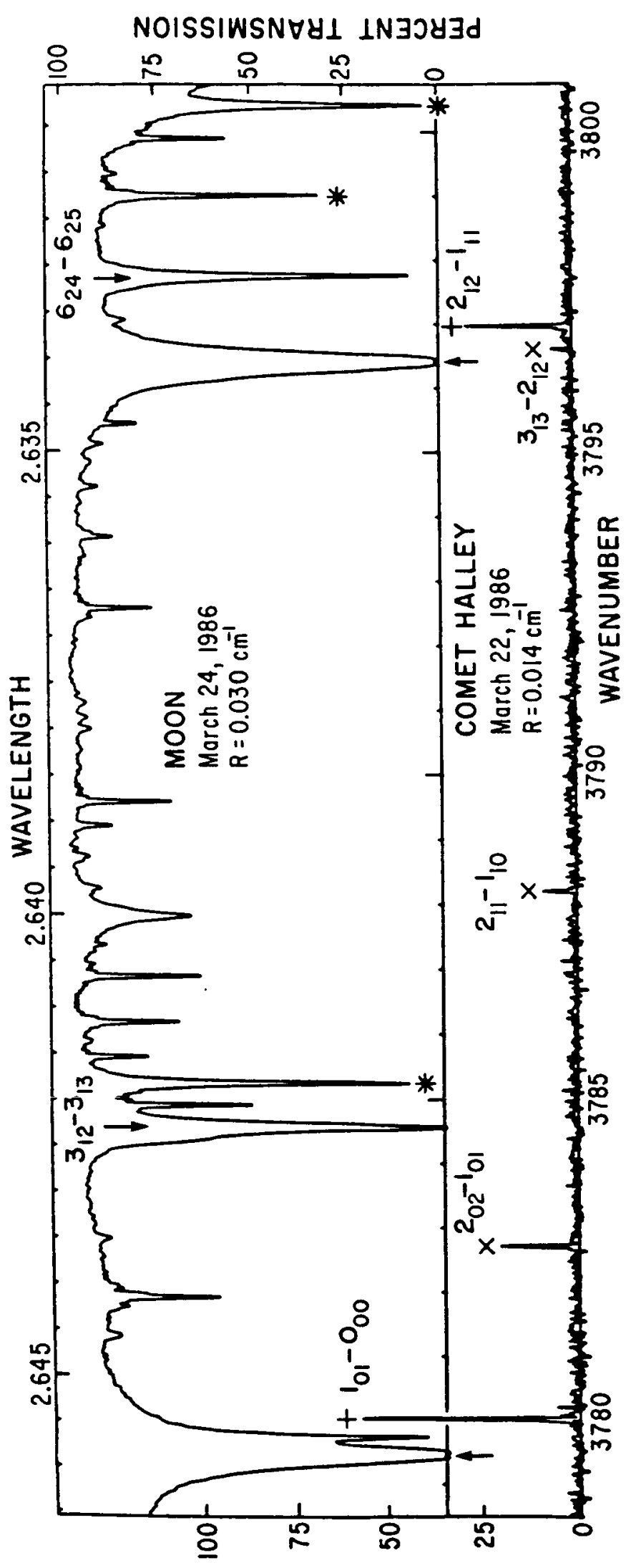

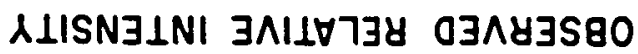

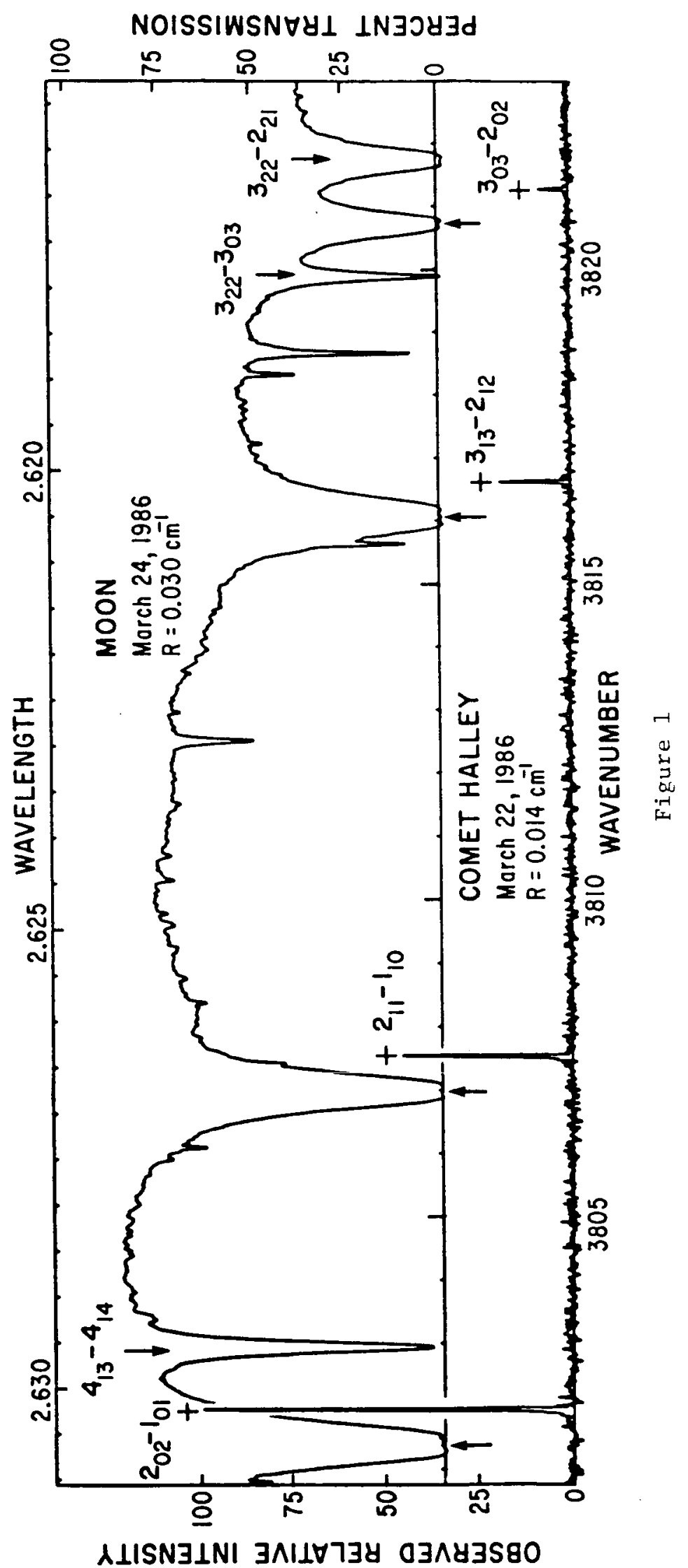




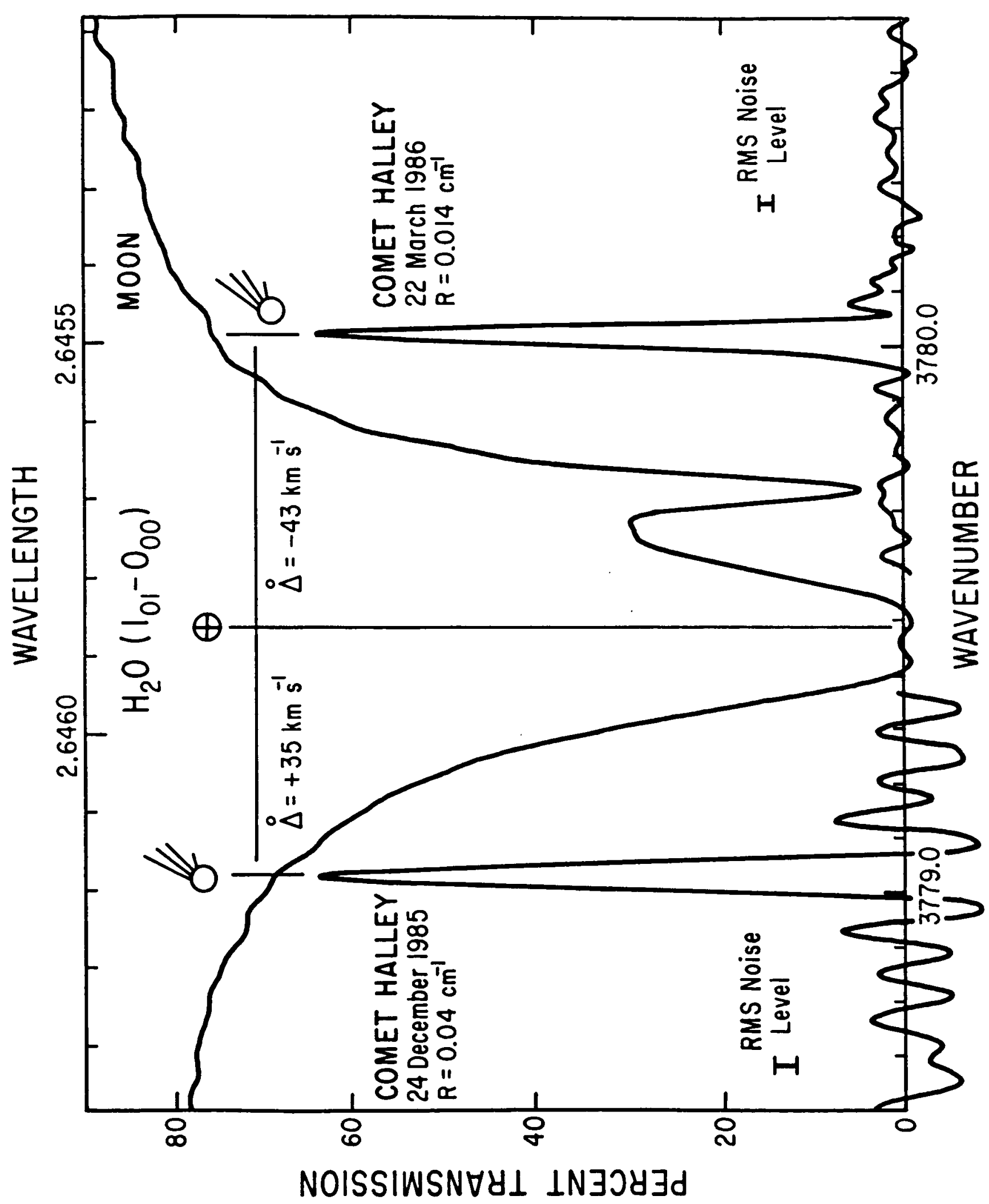




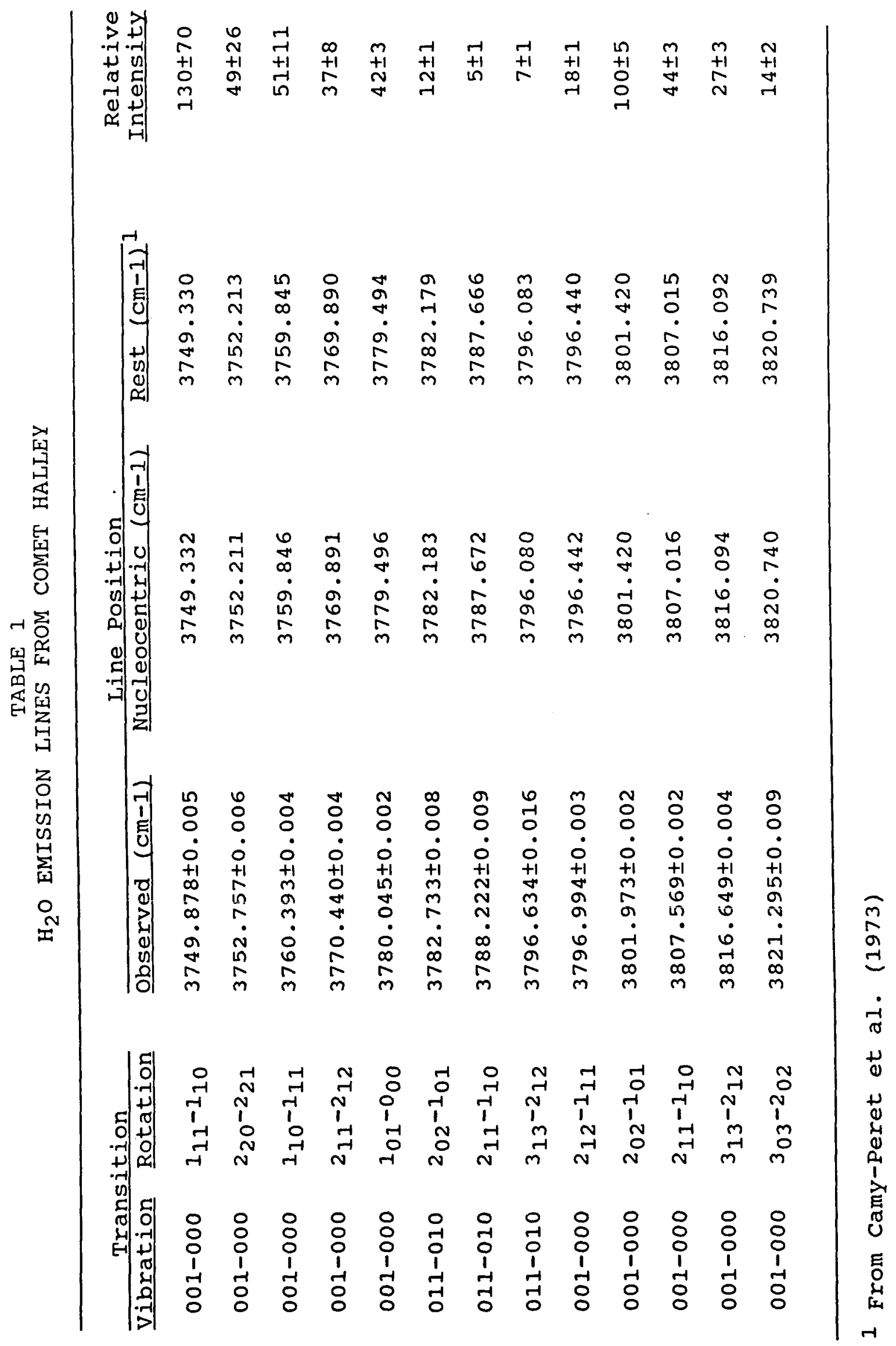




\begin{tabular}{|c|c|}
\hline $\begin{array}{l}\text { 1. Report No. } \\
\text { NASA TM-88367 }\end{array}$ & 3. Recipient's Catalog No. \\
\hline \multirow{2}{*}{$\begin{array}{l}\text { 4. Title and Subtitle } \\
\text { VELOCITY-RESOLVED OBSERVATIONS OF WATER IN COMET HALLEY }\end{array}$} & $\begin{array}{l}\text { 5. Report Date } \\
\text { September } 1986\end{array}$ \\
\hline & 6. Performing Organization Code \\
\hline \multirow{2}{*}{$\begin{array}{l}\text { 7. Author(s) } \\
\text { Harold P. Larson, * D. Scott Davis, } \dagger \text { Michael J. Mumma, } \neq \text { and } \\
\text { Harold A. Weaver } \xi\end{array}$} & $\begin{array}{l}\text { 8. Performing Organization Report No. } \\
\text { A-86410 }\end{array}$ \\
\hline & 10. Work Unit No. \\
\hline \multirow{3}{*}{$\begin{array}{l}\text { 9. Performing Organization Name and Address } \\
\text { *Lunar and Planetary Observatory, Univ. of Arizona, Tucson AZ } 85721 \text {, } \\
\text { tSteward Observatory, Univ. of Arizona, Tucson, AZ } 85721 \text {, } \\
\text { †Goddard Space Flight Center, Greenbelt, MD 20771, §Center for } \\
\text { Astrophysical Sciences, Johns Hopkins Univ., Baltimore, MD 20771 }\end{array}$} & \\
\hline & 11. Contract or Grant No. \\
\hline & 13. Type of Report and Period Covered \\
\hline \multirow{2}{*}{$\begin{array}{l}\text { 12. Sponsoring Agency Name and Address } \\
\text { National Aeronautics and Space Administration } \\
\text { Washington, DC } 20546\end{array}$} & Technical Memorandum \\
\hline & $\begin{array}{l}\text { 14. Sponsoring Agency Code } \\
352-02-03\end{array}$ \\
\hline \multicolumn{2}{|c|}{$\begin{array}{l}\text { 15. Supplementary Notes } \\
\text { Preprint Series \#58. Supported by NASA grants. } \\
\begin{array}{ll}\text { Point of Contact: } & \text { L. C. Haughney, Ames Research Center, MS 211-12, Moffett Field, CA } 94035 \\
& \text { (415) } 694-5339 \text { or FTS } 464-5339\end{array}\end{array}$} \\
\hline
\end{tabular}

16. Abstract

High resolution $\left(\lambda / \Delta \lambda \approx 3 \times 10^{5}\right)$ near-infrared observations of $\mathrm{H}_{2} \mathrm{O}$ emission from Comet Halley were acquired at the time of maximum post-perihelion geocentric Doppler shift. The observed widths and absolute positions of the $\mathrm{H}_{2} \mathrm{O}$ line profiles reveal characteristics of the molecular velocity field in the coma. These results support $\mathrm{H}_{2} \mathrm{O}$ outflow from a Sun-lit hemisphere or the entire nucleus, but not from a single, narrow jet emanating from the nucleus. The measured preand post-perihelion outflow velocities were $0.9 \pm 0.2$ and $1.4 \pm 0.2 \mathrm{~km} \mathrm{~s}^{-1}$, respectively. Temporal variations in the kinematic properties of the outflow were inferred from changes in the spectral line shapes. These results are consistent with the release of $\mathrm{H}_{2} \mathrm{O}$ into the coma from multiple jets.

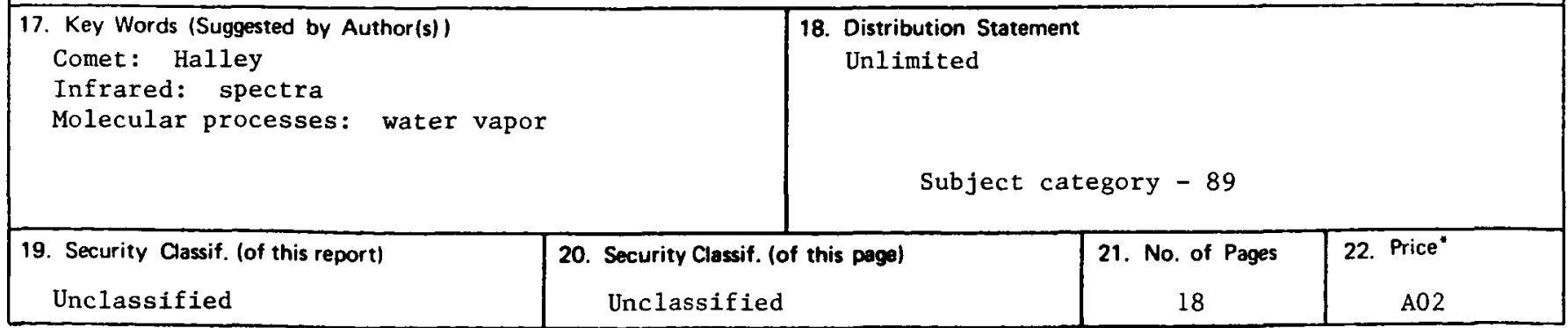

